\title{
Effect of local linear coupling on linear and nonlinear observables in circular accelerators
}

\author{
M. Hofer®* \\ CERN, CH-1211 Geneva, Switzerland and TU Wien, A-1040 Vienna, Austria \\ R. Tomás \\ CERN, CH-1211 Geneva, Switzerland
}

(Received 20 April 2020; accepted 8 September 2020; published 28 September 2020)

\begin{abstract}
Operation of particle accelerators and correction of unavoidable magnet or alignment errors critically depend on the assessment of both global and local observables such as tune or resonance driving terms. With most of the observables being a sum of different error sources, careful disentangling is necessary in order to establish an optimal correction and allow for smooth operation. In the LHC, linear coupling has been proven to have a major impact on the beam dynamics and is taken to be one of the main sources of uncertainty when establishing corrections. In this paper an approach to evaluate the change of the Hamiltonian terms with linear coupling is presented. The validity of derived equations is demonstrated on a number of observables and benchmarked against simulations.
\end{abstract}

DOI: 10.1103/PhysRevAccelBeams.23.094001

\section{INTRODUCTION}

Beam-based corrections of linear optics and nonlinear dynamics in current and future accelerator projects is of paramount importance to achieve their increasingly ambitious design goals. The evaluation of different error sources such as misalignment or magnetic field errors is based on measurements of either global quantities like the tune, chromaticity, and detuning terms or on local deviation from model values such as $\beta$-beating or resonance driving terms (RDT). In general, in the early commissioning phase of an accelerator focus is put on linear optics and coupling correction, both being major contributing factors in the performance of both hadron and lepton accelerators. Correction of linear coupling is usually based on either minimization of the global impact through reduction of the minimum possible tune separation or based on linear coupling RDTs or both.

The global effect of linear coupling is quantified by the closest tune approach $\Delta Q_{\min }$, that is, the minimum achievable distance between the fractional parts of the tunes [1]. More recently, this concept has been extended to include the effects of octupoles which lead to an amplitude dependence of the $\Delta Q_{\min }$ [2,3]. To identify sources of

*michael.hofer@cern.ch

Published by the American Physical Society under the terms of the Creative Commons Attribution 4.0 International license. Further distribution of this work must maintain attribution to the author(s) and the published article's title, journal citation, and DOI. linear coupling, the coupling RDTs $f_{1001}$ and $f_{1010}$ can be inferred from turn-by-turn data and serve as a basis for a local coupling correction. The relation between the coupling RDTs and $\Delta Q_{\min }$ can be found in [4]. While significant efforts have been made in recent years to improve measurement and correction techniques for linear (and nonlinear) coupling [5], the residual coupling left after corrections still retains an important role when describing the dynamics in the accelerator.

For example, in the LHC in late 2018, it was observed that while global coupling was well corrected, an erroneous local coupling bump in one interaction region had a significant impact on the beam size and thus on the luminosity [6,7]. Measurements of the $\beta$-function using the K-Modulation method did however not show any change in the tune shift generated from modulating the quadrupole gradient. Currently, no analytical derivation is available which may explain this insensitivity. A change of tune is not only used during the K-Modulation method, but also to determine nonlinear magnetic field errors in the triplet quadrupoles via feed down to a quadrupole gradient when changing the orbit in the quadrupoles [8,9].

Linear coupling is also assumed to be the single biggest source of uncertainty when trying to model the nonlinear behavior in the LHC. In [10], it was shown how nonlinear observables like detuning with amplitude are affected by linear coupling. This effect has been qualitatively demonstrated in [11] in simulations, where the impact of linear coupling on amplitude detuning is highlighted and how it may affect coherent instabilities in the LHC. Measurements of amplitude detuning are also used to establish corrections 
of nonlinear field errors in the triplet quadrupoles in the LHC [12]. Suboptimal correction of such may prove detrimental to beam lifetime and overall performance of the collider. To establish better corrections, the ability to determine the contribution of the local coupling to the measured amplitude detuning thus may prove quite helpful.

It was further shown in [10] that also measured RDTs change with linear coupling. In [13] it is shown how a skew sextupole resonance can be driven in the absence of any skew sextupole sources and away from the resonant condition due to interplay between the sextupoles and linear coupling. However, it is not apparent in this case how coupling would affect for example sextupole RDTs in the absence of skew sextupoles.

The aim of this paper is to quantify the effect that local linear coupling has on these global and local observables. Section II gives a brief introduction into the normal form formalism, concluding with a description of the particle motion under the influence of local coupling, as was already presented in [14]. In Sec. III, the coupled eigenvalues then allow us to express the effect on global and local observables under the influence of local coupling. This is then used in Sec. III A to show the impact on the change of tune during K-Modulation. The change of amplitude detuning generated by a single octupole in the presence of local coupling is treated in Sec. III C. Section III D then showcases the impact of local coupling on the RDT generated by a given multipole using as example sextupole RDTs.

\section{NORMAL FORM AND RESONANCE DRIVING TERM FORMALISM}

In circular accelerators, the one-turn motion of a single particle with the $4 \mathrm{D}$ coordinates $\mathbf{X}=\left(x, p_{x}, y, p_{y}\right)$ is described by

$$
\mathbf{X}_{s+C}=\mathcal{M}_{s} \mathbf{X}_{s}
$$

where $\mathcal{M}_{s}$ is the (sympletic) map of the accelerator lattice. For the sake of brevity, the dependence of the coordinates and following quantities on the longitudinal position $s$ is omitted in the following. For a lattice consisting of $W$ multipole elements the one-turn map is

$$
\mathcal{M}=M_{W+1} e^{: H_{W, n_{W}}}: M_{W} \ldots e^{: H_{2, n_{2}}:} M_{2} e^{: H_{1, n_{1}}}: M_{1},
$$

where $M_{w}$ is the linear transfer map of a given element $w$. $H_{w, n}$ is the Hamiltonian for a magnetic multipole as given by

$$
H_{w, n}=\frac{1}{n !} \Re\left[\left(K_{w, n}+i K_{w, n}^{S}\right)\left(x_{w}+i y_{w}\right)^{n}\right],
$$

with $e^{: H_{w, n}}$ : being the corresponding Lie-operator and $K_{w, n}$ and $K_{w, n}^{S}$ being the integrated magnetic strength of a normal and skew multipole, respectively. Here $n$ denotes the order of the multipole of the element $w$ with $n=2$ corresponding to a quadrupole. The transverse coordinates at the element $w$ are indicated with $x_{w}$ and $y_{w}$.

In the following, the resonance basis

$$
\mathbf{h}=\left(h_{x,+}, h_{x,-}, h_{y,+}, h_{y,-}\right)
$$

is used with the definition

$$
h_{q, \pm}=\hat{q} \pm i \hat{p}_{q}=\sqrt{2 J_{q}} e^{ \pm i \psi_{q}}, \quad q \in\{x, y\}
$$

where $\hat{q}$ and $\hat{p}_{q}$ are the normalized Courant-Snyder coordinates, defined as

$$
\left(\begin{array}{c}
\hat{q} \\
\hat{p}_{q}
\end{array}\right)=\left(\begin{array}{cc}
\frac{1}{\sqrt{\beta_{q}}} & 0 \\
\frac{\alpha_{q}}{\sqrt{\beta_{q}}} & \sqrt{\beta_{q}}
\end{array}\right)\left(\begin{array}{c}
q \\
p_{q}
\end{array}\right),
$$

and $J_{q}$ and $\psi_{q}$ the action and angle coordinates, respectively. The subscript ${ }_{w}$ for the coordinates, introduced in Eq. (3), has been omitted here and in the following as the information on the element in question is implicitly specified via the $s$-dependence of the $\beta$-functions.

Following the derivations in [15], the map $\mathcal{M}$ from Eq. (2) can be rewritten as

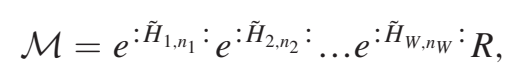

where $R$ is a rotation matrix. The Hamiltonian $\tilde{H}_{w, n}$ of a given element $w$ of order $n$ expressed in the resonance basis $\mathbf{h}$ is defined by

$$
\begin{aligned}
\tilde{H}_{w, n}= & \sum_{j k l m}^{n=j+k+l+m} h_{w, j k l m} e^{i\left[(j-k) \Delta \phi_{w, x}+(l-m) \Delta \phi_{w, y}\right]} \\
& \times h_{x,+}^{j} h_{x,-}^{k} h_{y,+}^{l} h_{y,-}^{m},
\end{aligned}
$$

where $\Delta \phi_{w, x}$ and $\Delta \phi_{w, y}$ are the phase advances from the element $w$ to an observation point in the horizontal and vertical plane, respectively. The coefficient $h_{w, j k l m}$ is expressed by

$$
h_{w, j k l m}=\frac{\Re\left(i^{l+m}\left[K_{w, n}+i K_{w, n}^{S}\right]\right)}{j ! k ! l ! m ! 2^{n}}\left(\beta_{w, x}\right)^{\frac{j+k}{2}}\left(\beta_{w, y}\right)^{\frac{l+m}{2}} .
$$

Using the Baker-Campbell-Hausdorff formula

$$
e^{A} e^{B}=e^{A+B+\frac{1}{2}[A, B]+\cdots}
$$

Eq. (7) can be expressed as 


$$
\begin{aligned}
& e^{: \tilde{H}_{1, n_{1}}}: e^{: \tilde{H}_{2, n_{2}}}: \ldots e^{: \tilde{H}_{W, n_{W}}}: \\
& \approx e^{\sum_{w}^{W} \tilde{H}_{w, n_{w}}+\frac{1}{2} \sum_{w}^{W} \sum_{v}^{w-1}\left[\tilde{H}_{w, n_{w}}, \tilde{H}_{v, n_{v}}\right]+\cdots},
\end{aligned}
$$

where $\cdots$ indicates terms of order 3 and higher. In the normal form approach, the basis $\mathbf{h}$ is then transformed into the new basis $\zeta$ following [16]

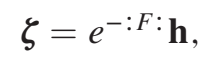

using a polynomial generating function

$$
F=\sum_{j k l m} f_{j k l m} \zeta_{x,+}^{j} \zeta_{x,-}^{k} \zeta_{y,+}^{l} \zeta_{y,-}^{m},
$$

where $\zeta_{q, \pm}=\sqrt{2 I_{q}} e^{ \pm i \phi_{q}}$ and $f_{j k l m}$ denotes the resonance driving term.

It can be shown that the RDT $f_{j k l m}$ in first order is

$$
f_{j k l m}=\frac{\sum_{w} h_{w, j k l m} e^{i\left[(j-k) \Delta \phi_{w, x}+(l-m) \Delta \phi_{w, y}\right]}}{1-e^{2 \pi i\left[(j-k) Q_{x}+(l-m) Q_{y}\right]}},
$$

where $h_{w, j k l m}$ from Eq. (9) is used, which is obtained when approximating Eq. (11) as

$$
e^{: \tilde{H}_{1, n_{1}}:} e^{: \tilde{H}_{2, n_{2}}: \ldots e^{: \tilde{H}_{W, n_{W}}:}} \approx e^{\sum_{w}^{W} \tilde{H}_{W, n_{W}}} .
$$

The expansion to second order can be found in $[13,15]$.

\section{A. Particle motion under the influence of coupling}

Following Eq. (12), the transformation between normal form coordinates and Courant-Snyder coordinates reads

$$
\begin{aligned}
& h_{q, \pm}=\hat{q} \pm i \hat{p}_{q}=\sqrt{2 J_{q}} e^{ \pm i \psi_{q}}
\end{aligned}
$$

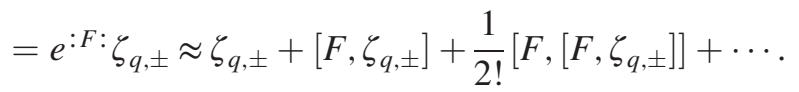

In [14], it was shown that for the case of taking into account only linear perturbations such as either $\beta$-beating or linear coupling, a closed form of this transformation exists. In the case of linear coupling, the generating function $F$ reads

$$
\begin{aligned}
F= & f_{1001} \zeta_{x,+} \zeta_{y,-}+f_{1010} \zeta_{x,+} \zeta_{y,+} \\
& +f_{1001}^{*} \zeta_{x,-} \zeta_{y,+}+f_{1010}^{*} \zeta_{x,-} \zeta_{y,-}
\end{aligned}
$$

where the RDT $f_{1001}$ drives the difference resonance $\left(Q_{x}-Q_{y}\right)=p$ and $f_{1010}$ corresponds to the sum resonance $\left(Q_{x}+Q_{y}\right)=p$. Notably, this generating function only accounts for the geometric distortion due to coupling resonances and not for any change due to terms like $f_{2000}$ which are excited by second and higher order interplay between skew quadrupoles. Using Eq. (16) together with the generating function F from Eq. (17) and

$$
\left[\zeta_{q,+}^{u}, \zeta_{q,-}\right]=-2 i u \zeta_{q,+}^{u-1},
$$

it is shown in [14] that the components of the resonance basis $\mathbf{h}$ can be expressed as

$$
\begin{aligned}
& h_{x,-}=\zeta_{x,-} \cosh 2 \mathcal{P}-i \frac{\sinh 2 \mathcal{P}}{\mathcal{P}}\left(f_{1001} \zeta_{y,-}+f_{1010} \zeta_{y,+}\right) \\
& h_{x,+}=\zeta_{x,+} \cosh 2 \mathcal{P}+i \frac{\sinh 2 \mathcal{P}}{\mathcal{P}}\left(f_{1010}^{*} \zeta_{y,-}+f_{1001}^{*} \zeta_{y,+}\right) \\
& h_{y,-}=\zeta_{y,-} \cosh 2 \mathcal{P}-i \frac{\sinh 2 \mathcal{P}}{\mathcal{P}}\left(f_{1001}^{*} \zeta_{x,-}+f_{1010} \zeta_{x,+}\right) \\
& h_{y,+}=\zeta_{y,+} \cosh 2 \mathcal{P}+i \frac{\sinh 2 \mathcal{P}}{\mathcal{P}}\left(f_{1010}^{*} \zeta_{x,-}+f_{1001} \zeta_{x,+}\right)
\end{aligned}
$$

where $2 \mathcal{P}=\sqrt{\left|2 f_{1010}\right|^{2}-\left|2 f_{1001}\right|^{2}}$. The particle coordinate $q$ is given by

$$
q=\frac{\sqrt{\beta_{q}}}{2}\left(h_{q,+}+h_{q,-}\right) .
$$

Using Eq. (19) and Eq. (20) the particles coordinates now read

$$
\begin{aligned}
& x=\frac{\sqrt{\beta_{x}}}{2}\left[\zeta_{x,+} \mathcal{C}+\zeta_{x,-} \mathcal{C}+i \zeta_{y,+} \hat{F}_{y x}^{*}-i \zeta_{y,-} \hat{F}_{y x}\right], \\
& y=\frac{\sqrt{\beta_{y}}}{2}\left[\zeta_{y,+} \mathcal{C}+\zeta_{y,-} \mathcal{C}+i \zeta_{x,+} \hat{F}_{x y}^{*}-i \zeta_{x,-} \hat{F}_{x y}\right],
\end{aligned}
$$

where the superscript ${ }^{*}$ denotes the complex conjugate and

$$
\begin{gathered}
\mathcal{C}=\cosh 2 \mathcal{P}, \\
\hat{F}_{y x}=\frac{\sinh 2 \mathcal{P}}{\mathcal{P}}\left(f_{1001}^{*}-f_{1010}^{*}\right), \\
\hat{F}_{x y}=\frac{\sinh 2 \mathcal{P}}{\mathcal{P}}\left(f_{1001}-f_{1010}^{*}\right)
\end{gathered}
$$

have been introduced. Equations (23) and (24) represent the combined coupling RDTs presented in [13] scaled by a factor $\frac{\sinh 2 \mathcal{P}}{\mathcal{P}}$. In case of $\left|f_{1001}\right|=\left|f_{1010}\right|, \mathcal{C}$ and $\frac{\sinh 2 \mathcal{P}}{\mathcal{P}}$ reduce to 1 and 2, respectively. It should be noted that in the following section for the benchmarking of the derivations, the coupling RDTs $f_{1001}$ and $f_{1010}$ have been evaluated by expressing them as functions of the coupling matrix, as is shown in [17]. This way, all orders of the coupling RDTs are also taken into account and due to the closed form of the transformation (16), by using the generating function from Eq. (17), also the transformation is accurate to arbitrary order. 


\section{CHANGE OF OBSERVABLES DUE TO COUPLING}

In [15], contributions to tune and amplitude detuning up to second order have been derived. Similarly so, in [13], resonance driving term coefficients up to second order are presented. In both cases, it is apparent that the first order contributions from e.g., octupole to amplitude detuning is not modified in the presence of coupling and changes stem from other contributions such as skew octupoles in conjunction with coupling. Yet simulation work presented in [11] clearly shows that amplitude detuning generated by octupoles is affected in the presence of local coupling, also in the absence of other skew elements.

These findings indicate that the observed effect could stem from third order contributions or higher. As higher order expansion become more and more cumbersome, here an alternative approach is presented, which is specifically applicable to linear coupling. Using the coupled coordinates from Eq. (21), the Hamiltonian from Eq. (3) can be expanded leading to

$$
\begin{aligned}
& \tilde{H}_{w, n}^{c}=\frac{1}{n !} \frac{1}{2}\left\{\left(K_{w, n}+i K_{w, n}^{S}\right)\right. \\
& \times\left[\zeta_{x,+}\left(\frac{\sqrt{\beta_{x}}}{2} \mathcal{C}-\frac{\sqrt{\beta_{y}}}{2} \hat{F}_{x y}^{*}\right)+\zeta_{x,-}\left(\frac{\sqrt{\beta_{x}}}{2} \mathcal{C}+\frac{\sqrt{\beta_{y}}}{2} \hat{F}_{x y}\right)\right. \\
& \left.+i \zeta_{y,+}\left(\frac{\sqrt{\beta_{y}}}{2} \mathcal{C}+\frac{\sqrt{\beta_{x}}}{2} \hat{F}_{y x}^{*}\right)+i \zeta_{y,-}\left(\frac{\sqrt{\beta_{y}}}{2} \mathcal{C}-\frac{\sqrt{\beta_{x}}}{2} \hat{F}_{y x}\right)\right]^{n} \\
& +\left(K_{w, n}-i K_{w, n}^{S}\right) \\
& \times\left[\zeta_{x,-}\left(\frac{\sqrt{\beta_{x}}}{2} \mathcal{C}-\frac{\sqrt{\beta_{y}}}{2} \hat{F}_{x y}\right)+\zeta_{x,+}\left(\frac{\sqrt{\beta_{x}}}{2} \mathcal{C}+\frac{\sqrt{\beta_{y}}}{2} \hat{F}_{x y}^{*}\right)\right. \\
& \left.\left.-i \zeta_{y,-}\left(\frac{\sqrt{\beta_{y}}}{2} \mathcal{C}+\frac{\sqrt{\beta_{x}}}{2} \hat{F}_{y x}\right)-i \zeta_{y,+}\left(\frac{\sqrt{\beta_{y}}}{2} \mathcal{C}-\frac{\sqrt{\beta_{x}}}{2} \hat{F}_{y x}^{*}\right)\right]^{n}\right\},
\end{aligned}
$$

where $\Re(z)=\frac{z+z^{*}}{2}$ and $\zeta_{q, \pm}^{*}=\zeta_{q, \mp}$ has been used. Here the superscript ${ }^{c}$ has been introduced as to distinguish from the Hamiltonian using the uncoupled basis. Following the multinomial theorem

$$
\begin{aligned}
& \left(x_{1}+x_{2}+\cdots+x_{m}\right)^{n} \\
& \quad=\sum_{k_{1}+k_{2}+\cdots k_{m}=n} \frac{n !}{k_{1} ! k_{2} ! \ldots k_{m} !} \prod_{t=1}^{m} x_{t}^{k_{t}}
\end{aligned}
$$

the Hamiltonian is rewritten as

$$
\begin{aligned}
\tilde{H}_{w, n}^{c}= & \sum_{j k l m}^{n=j+k+l+m}\left[\frac{i^{l+m}\left(K_{w, n}+i K_{w, n}^{S}\right)^{j}}{j ! k ! l ! m ! 2^{n+1}} \zeta_{x,+}^{j k} \zeta_{x,-}^{k l} \zeta_{y,+}^{l} \zeta_{y,-}^{m}\right. \\
& \times\left(\sqrt{\beta_{x}} \mathcal{C}-\sqrt{\beta_{y}} \hat{F}_{x y}^{*}\right)^{j}\left(\sqrt{\beta_{x}} \mathcal{C}+\sqrt{\beta_{y}} \hat{F}_{x y}\right)^{k} \\
& \left.\times\left(\sqrt{\beta_{y}} \mathcal{C}+\sqrt{\beta_{x}} \hat{F}_{y x}^{*}\right)^{l}\left(\sqrt{\beta_{y}} \mathcal{C}-\sqrt{\beta_{x}} \hat{F}_{y x}\right)^{m}\right] \\
& +\left[\frac{(-i)^{l+m}\left(K_{w, n}-i K_{w, n}^{S}\right)^{j}}{j ! k ! l ! m ! 2^{n+1}} \zeta_{x,-}^{k} \zeta_{y,+}^{l} \zeta_{y,-}^{m}\right. \\
& \times\left(\sqrt{\beta_{x}} \mathcal{C}+\sqrt{\beta_{y}} \hat{F}_{x y}^{*}\right)^{j}\left(\sqrt{\beta_{x}} \mathcal{C}-\sqrt{\beta_{y}} \hat{F}_{x y}\right)^{k} \\
& \left.\times\left(\sqrt{\beta_{y}} \mathcal{C}-\sqrt{\beta_{x}} \hat{F}_{y x}^{*}\right)^{l}\left(\sqrt{\beta_{y}} \mathcal{C}+\sqrt{\beta_{x}} \hat{F}_{y x}\right)^{m}\right]
\end{aligned}
$$

It follows that the coefficient $h_{w, j k l m}^{c}$ from Eq. (9) in the presence of coupling can then be rewritten as

$$
\begin{aligned}
h_{w, j k l m}^{c}= & \frac{i^{l+m}\left[K_{w, n}+i K_{w, n}^{S}\right]}{j ! k ! l ! m ! 2^{n+1}} \\
& \times\left[\left(\sqrt{\beta_{x}} \mathcal{C}-\sqrt{\beta_{y}} \hat{F}_{x y}^{*}\right)^{j}\left(\sqrt{\beta_{x}} \mathcal{C}+\sqrt{\beta_{y}} \hat{F}_{x y}\right)^{k}\right. \\
& \left.\times\left(\sqrt{\beta_{y}} \mathcal{C}+\sqrt{\beta_{x}} \hat{F}_{y x}^{*}\right)^{l}\left(\sqrt{\beta_{y}} \mathcal{C}-\sqrt{\beta_{x}} \hat{F}_{y x}\right)^{m}\right] \\
& +\frac{(-i)^{l+m}\left[K_{w, n}-i K_{w, n}^{S}\right]}{j ! k ! l ! m ! 2^{n+1}} \\
& \times\left[\left(\sqrt{\beta_{x}} \mathcal{C}+\sqrt{\beta_{y}} \hat{F}_{x y}^{*}\right)^{j}\left(\sqrt{\beta_{x}} \mathcal{C}-\sqrt{\beta_{y}} \hat{F}_{x y}\right)^{k}\right. \\
& \left.\times\left(\sqrt{\beta_{y}} \mathcal{C}-\sqrt{\beta_{x}} \hat{F}_{y x}^{*}\right)^{l}\left(\sqrt{\beta_{y}} \mathcal{C}+\sqrt{\beta_{x}} \hat{F}_{y x}\right)^{m}\right]
\end{aligned}
$$

and similar so to obtain $f_{j k l m}^{c}$ following Eq. (14). In the following, only the first order in strength of the natural source will be used in the evaluation of the Hamiltonian, however, a higher-order expansion analogously to [13] is possible as well. It should also be mentioned that by including a dispersion term $D_{q} \delta_{p}$ in the coupled coordinates from Eq. (21), also the behavior of the off-momentum Hamiltonian can be studied.

\section{A. Impact of local coupling on K-Modulation}

As a first application of the previous derivation, the impact of local coupling on the tune change from a change in quadrupole is examined. This change of tune is for example used during K-Modulation to determine the average $\beta$-function in quadrupoles, but has also been used in the LHC to determine nonlinear field errors in the triplet quadrupoles by changing the orbit and observing the tune change due to feed down. In general, the tune shift for a given Hamiltonian $\tilde{H}$ is 


$$
\Delta Q_{q}=\frac{1}{2 \pi} \frac{\partial\langle\tilde{H}\rangle}{\partial J_{q}}
$$

where $\langle\tilde{H}\rangle$ is the Hamiltonian average over the phase variables. As such, only phase-independent terms in the Hamiltonian will contribute. In the case of a horizontal (vertical) tune change for a quadrupole, this term corresponds to $h_{1100}\left(h_{0011}\right)$.

Taking the Hamilton from Eq. (27) for the case of a thin quadrupole $(n=2)$ together with Eq. (29), the tune shift in the presence of local coupling is then

$$
\begin{aligned}
& \Delta Q_{x}^{c}=\frac{\Delta K_{2}}{4 \pi}\left[\beta_{x} \mathcal{C}^{2}-\beta_{y}\left|\hat{F}_{x y}\right|^{2}\right], \\
& \Delta Q_{y}^{c}=-\frac{\Delta K_{2}}{4 \pi}\left[\beta_{y} \mathcal{C}^{2}-\beta_{x}\left|\hat{F}_{y x}\right|^{2}\right],
\end{aligned}
$$

where the subscript ${ }_{w}$ has been omitted as here the case for only one element is examined. From this equation, it is apparent that any local coupling will tend to result in a decrease of the induced tune change.

We note that in the case of no local coupling $\left(f_{1001}=\right.$ $f_{1010}=0$ ), these formulas reduce to the case of a thin quadrupole excitation

$$
\Delta Q_{x, y}^{\text {approx }}= \pm \frac{\Delta K_{2} \beta_{x, y}}{4 \pi},
$$

which is an approximation of

$$
\begin{aligned}
\Delta Q_{x, y}^{\text {exact }}= & -Q_{x, y}+\frac{1}{2 \pi} \arccos \left[\cos \left(2 \pi Q_{x, y}\right)\right. \\
& \left.\mp \frac{\beta_{x, y} \Delta K_{2}}{2} \sin \left(2 \pi Q_{x, y}\right)\right],
\end{aligned}
$$

the approximation being valid in case of a small excitation $\Delta K_{2}$ and sufficiently far from the integer and half-integer resonance [18]. To overcome these limitations, the first part of Eq. (30) can be replaced by Eq. (32) yielding

$$
\begin{aligned}
& \Delta Q_{x}^{c}=\Delta Q_{x}^{\text {exact }} \mathcal{C}^{2}-\frac{\Delta K_{2}}{4 \pi} \beta_{y}\left|\hat{F}_{x y}\right|^{2} \\
& \Delta Q_{y}^{c}=\Delta Q_{y}^{\text {exact }} \mathcal{C}^{2}+\frac{\Delta K_{2}}{4 \pi} \beta_{x}\left|\hat{F}_{y x}\right|^{2} .
\end{aligned}
$$

We note that both Eq. (30) and (33) still only include the first-order contribution of $\Delta K_{2}$ to tune shift, which is only exact in case of a thin quadrupole. In [19], a derivation is presented including the second-order contribution in $\Delta K_{2}$ in case of a thick quadrupole.

To check the validity of the derived formulas and impact of higher-order contributions, MAD-X simulations were conducted using the LHC lattice. To mitigate interference from effects such as changes in the $\beta$-function through thick elements, a lattice composed of thin (zero length) elements was used.
For the conducted studies the inner triplet of the experimental insertion in LHC Point 1 was used. The location was chosen as in the LHC the $\beta$-function at the interaction point $\left(\beta^{*}\right)$ is usually inferred from K-Modulation, a method which allows to calculate the average $\beta$-function in a quadrupole by measuring the tune change $\Delta Q$ under a modulation of the quadrupole gradient $\Delta K_{2}$.

A change of this $\Delta Q$ due to local linear coupling at the location of the quadruple may thus affect the accuracy with which $\beta^{*}$ can be reconstructed.

It is also here where due to the large $\beta$-function, an erroneous rotation of a triplet quadrupole around the longitudinal axis may give rise to a considerable skew quadrupole component. A closed coupling bump is created by using the skew quadrupole correctors located between the second and third quadrupole of the final focus triplet left and right of the interaction point (IP), as illustrated in Fig. 1.

Due to the phase difference between the left and right skew quadrupole corrector being close to $180^{\circ}$, and if powered with opposite strength, the perturbation of the first skew quadrupole is canceled by the second one and $\Delta Q_{\min }$ is marginally affected. After application of the skew quadrupole correctors, the tunes were rematched to the original values of $Q_{x}=0.28$ and $Q_{y}=0.31$ to compensate for the small second-order contribution of the correctors to tune. In this configuration however, the two coupling terms cannot be controlled independently and $\left|f_{1010}\right|=\left|f_{1001}\right|$.

The gradient of one slice of the first quadrupole right of the interaction point was changed to induce a tune change in the uncoupled case of $\approx 0.01$ in the plane with the larger $\beta$-function. The sign of the gradient change was chosen such that the tunes drift apart to mitigate any interference of a possible closest tune approach. A comparison between both formulas to results from MAD-X simulations is presented in Fig. 2. We note that Eq. (30) presents an offset even in case of no coupling. This offset is attributed to the use of the approximation Eq. (31) in Eq. (30). Equation (33), which does not rely on this approximation, does not present this offset anymore and shows good agreement for the probed levels of coupling. In Fig. 3 the reduction in percent is presented for different ratios of $\frac{\beta_{x}}{\beta_{y}}$. Notably, for an amplitude of $\left|f_{1010}\right|$ around 0.15, the change of tune is below $0.1 \%$ in both planes with respect to the uncoupled case.

\section{B. Effect on beam size}

In late 2018, it was observed that a strong local coupling in the interaction point 2 in the LHC, introduced by an erroneous swap of two corrector settings, lead to a reduction of the luminosity of about a factor $2[6,7]$. In the previous section, it was shown that even for a sizable local coupling, no significant change in $\Delta Q$ is observed. As such, K-Modulation measurements are only weakly 

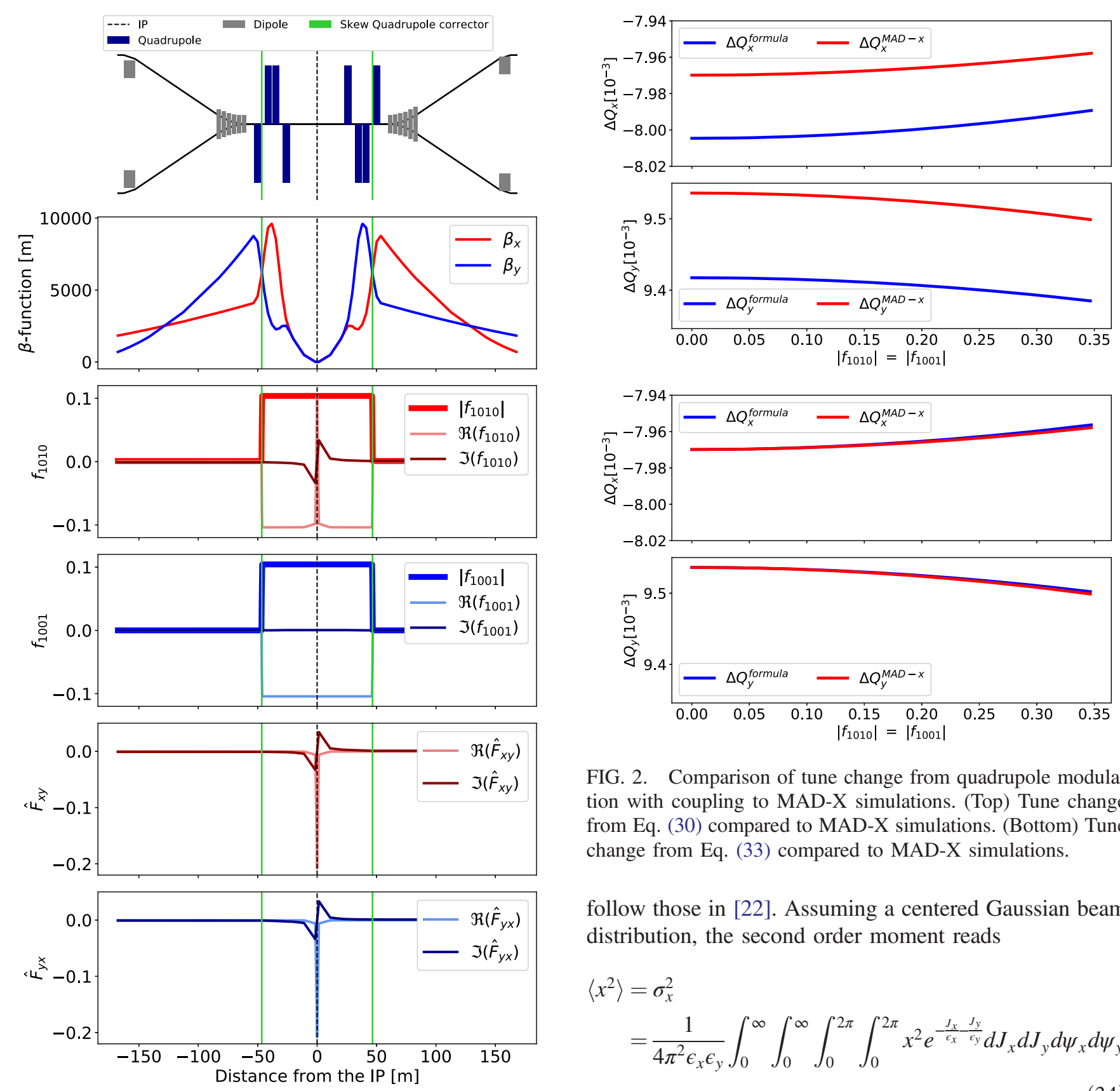

FIG. 1. Illustration of a LHC interaction region. The optics presented here corresponds to $\beta^{*}=25 \mathrm{~cm}$ and the strength of the skew quadrupole correctors (shown in green) was set to $K_{2}^{S} L=6.7 \times 10^{-5} \mathrm{~m}^{-2}$.

affected by the presence of local coupling, which has also been demonstrated in a dedicated experiment in the LHC [20]. On the other hand, tracking studies presented in [21] show a drastic increase of the beam size at the IP if the local coupling bump is introduced, able to explain most of observed luminosity loss.

In the following, it will be shown how for the same setup as used for the tune change a significant increase in beam size can be calculated. The derivations presented here
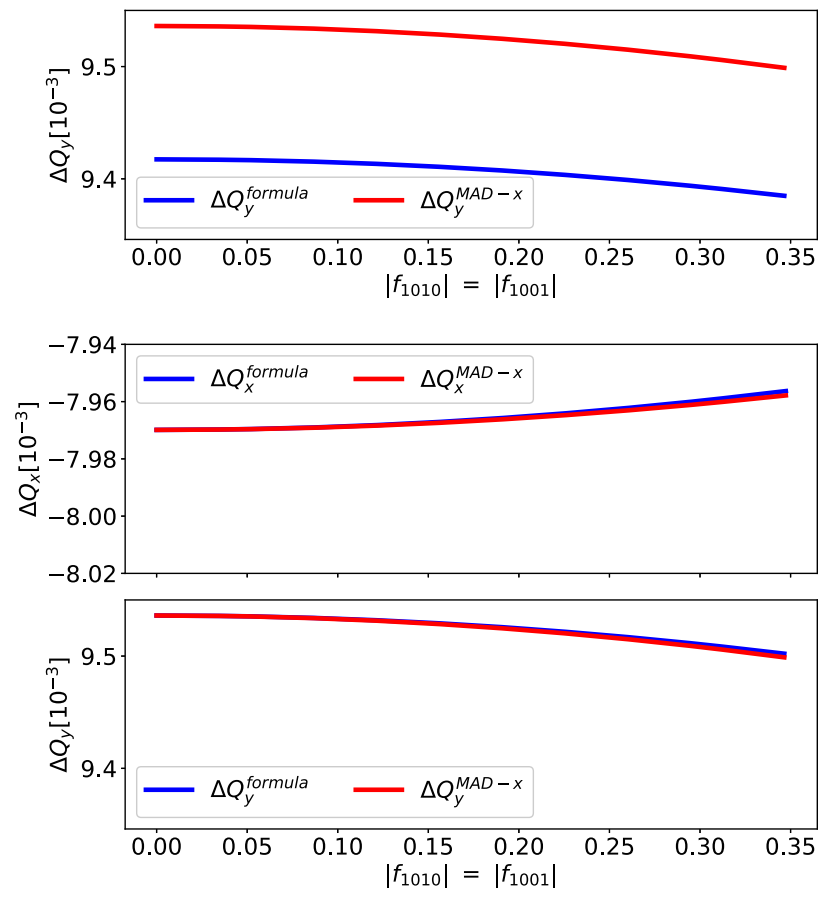

FIG. 2. Comparison of tune change from quadrupole modulation with coupling to MAD-X simulations. (Top) Tune change from Eq. (30) compared to MAD-X simulations. (Bottom) Tune change from Eq. (33) compared to MAD-X simulations.

follow those in [22]. Assuming a centered Gaussian beam distribution, the second order moment reads

$$
\begin{aligned}
\left\langle x^{2}\right\rangle & =\sigma_{x}^{2} \\
& =\frac{1}{4 \pi^{2} \epsilon_{x} \epsilon_{y}} \int_{0}^{\infty} \int_{0}^{\infty} \int_{0}^{2 \pi} \int_{0}^{2 \pi} x^{2} e^{-\frac{J_{x}}{\epsilon_{x}}-\frac{J_{y}}{\epsilon_{y}}} d J_{x} d J_{y} d \psi_{x} d \psi_{y}
\end{aligned}
$$

where $\epsilon_{x, y}$ is the uncoupled RMS emittance in the horizontal and vertical plane, respectively. Using Eq. (21) one then obtains

$$
\sigma_{x}^{c}=\sqrt{\left\langle x^{2}\right\rangle}=\left[\beta_{x} \epsilon_{x} \mathcal{C}^{2}+\beta_{x} \epsilon_{y}\left|\hat{F}_{y x}\right|^{2}\right]^{\frac{1}{2}} .
$$

Note that here the emittance exchange effect on the RMS emittances, described in [14] using the RDT formalism, is not taken into account. In a similar fashion, the beam size in the vertical plane reads

$$
\sigma_{y}^{c}=\sqrt{\left\langle y^{2}\right\rangle}=\left[\beta_{y} \epsilon_{y} \mathcal{C}^{2}+\beta_{y} \epsilon_{x}\left|\hat{F}_{x y}\right|^{2}\right]^{\frac{1}{2}} .
$$



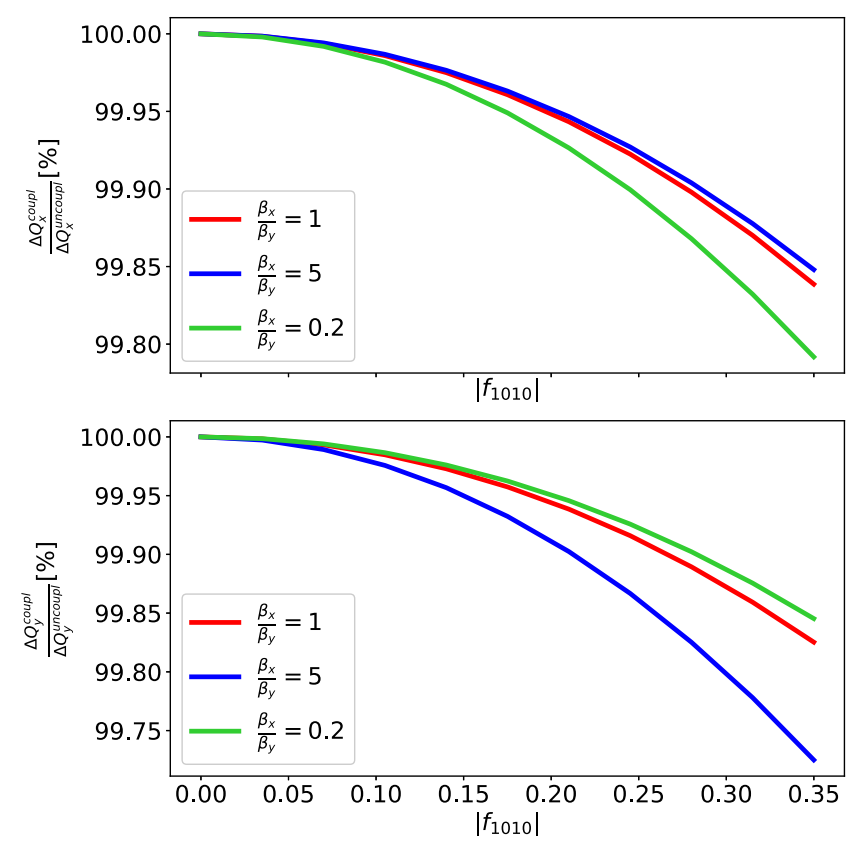

FIG. 3. Change of the induced tune change from change of quadrupole gradient with local coupling amplitude.

Local coupling will not only result in an increase of beamsize but can also introduce a tilt angle in the $x-y-p l a n e$, as shown in [23]. The tilt angle $\psi$ relates to the elements of the sigma matrix via

$$
\tan (2 \psi)=\frac{2\langle x y\rangle}{\left\langle x^{2}\right\rangle-\left\langle y^{2}\right\rangle}
$$

where

$$
\langle x y\rangle=\left[\sqrt{\beta_{x} \beta_{y}} \mathcal{C}\left(\epsilon_{x} \Im \hat{F}_{x y}+\epsilon_{y} \Im \hat{F}_{y x}\right)\right]
$$

is used.

A comparison of the increase in beam size in the IP predicted by the formula with the MAD-X tracking simulations, using the setup described in the previous section, is presented in Fig. 4. For this tracking studies 10000 particles were tracked for 256 turns using MAD-X. Notably, for $\left|f_{1010}\right|$ in a range where a tune change of below $0.1 \%$ is observed, the beam size at the IP can increase by almost $20 \%$ for equal emittances in both planes.

Observing Fig. 1, one can see that due to the jump of the $f_{1010}$ coupling RDT and in the following also $\hat{F}_{x y}$ and $\hat{F}_{y x}$ at the IP, the local coupling leads to a large effect on the beam size at this position. On the other hand, $\hat{F}_{x y}$ and $\hat{F}_{y x}$ at the position of the innermost quadrupoles are significantly lower, thus explaining the small impact on the $\mathrm{K}$-modulation measurements.

Based on these formulas, the beam size inferred from optics measurements can be corrected to accurately
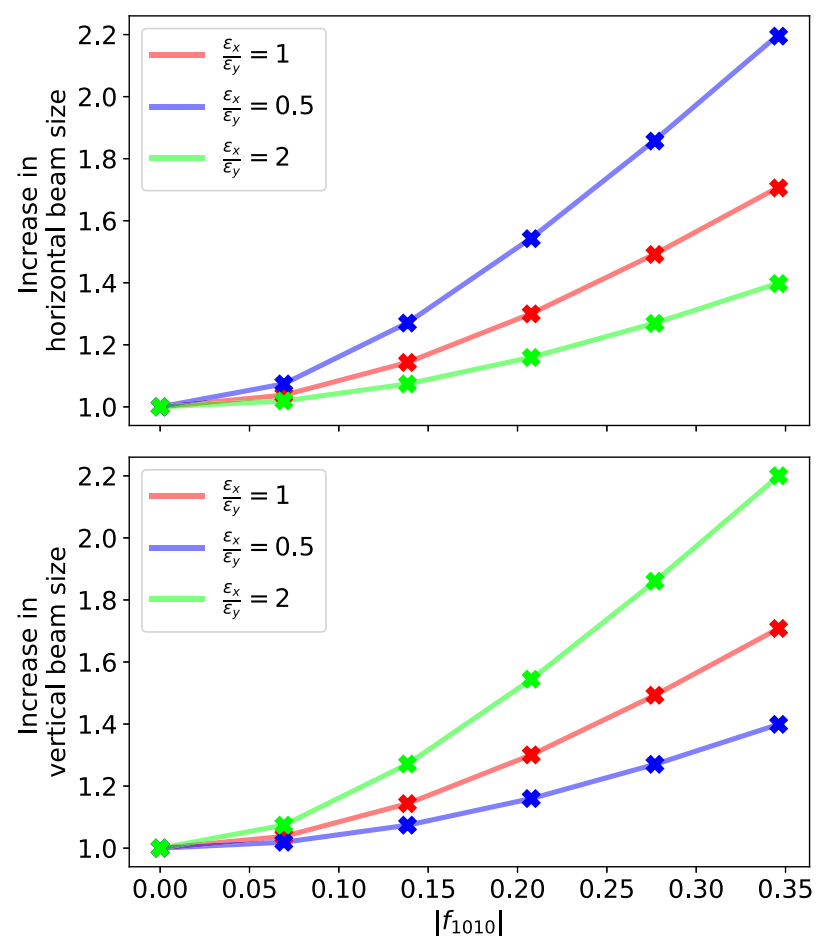

FIG. 4. Increase of beam size in IP1 as a function of the absolute value of the coupling RDT.

represent the real beam size if local coupling is evaluated by other means. This could for example be used to correct the inferred emittance based on beam size measurements provided by instruments such as synchrotron light monitors.

\section{Impact on amplitude detuning generated by octupoles}

A second global observable of particular importance in the LHC is the detuning with amplitude generated by octupoles due to its role in damping coherent instabilities. Previous studies presented in [11] have already shown how linear coupling may lead to a loss of Landau damping. However, no analytical relation between local coupling and the decrease in amplitude detuning has been established.

Similar to the derivation of the change of tune presented in the previous section, we derive here equations to describe the impact of coupling on the amplitude detuning generated by octupoles. The amplitude detuning for a given Hamiltonian $\tilde{H}$ is calculated by

$$
\frac{\partial Q_{q}}{\partial J_{u}}=\frac{1}{2 \pi} \frac{\partial^{2}\langle\tilde{H}\rangle}{\partial J_{q} \partial J_{u}}
$$

Using the previous equation together with the Hamiltonian from Eq. (27) for the case of a thin octupole, the direct and 
cross-plane amplitude detuning terms in the presence of linear coupling read

$$
\begin{aligned}
\frac{\partial Q_{x}^{c}}{\partial J_{x}}= & \frac{K_{4}}{16 \pi}\left[\beta_{x}^{2} \mathcal{C}^{4}+\beta_{y}^{2}\left|\hat{F}_{x y}\right|^{4}\right. \\
& \left.-2 \beta_{x} \beta_{y} \mathcal{C}^{2}\left(\left|\hat{F}_{x y}\right|^{2}+2\left(\Im \hat{F}_{x y}\right)^{2}\right)\right], \\
\frac{\partial Q_{y}^{c}}{\partial J_{y}}= & \frac{K_{4}}{16 \pi}\left[\beta_{y}^{2} \mathcal{C}^{4}+\beta_{x}^{2}\left|\hat{F}_{y x}\right|^{4}\right. \\
& \left.-2 \beta_{x} \beta_{y} \mathcal{C}^{2}\left(\left|\hat{F}_{y x}\right|^{2}+2\left(\Im \hat{F}_{y x}\right)^{2}\right)\right], \\
\frac{\partial Q_{x}^{c}}{\partial J_{y}}=\frac{\partial Q_{y}^{c}}{\partial J_{x}}= & \frac{K_{4}}{8 \pi}\left[\beta_{x}^{2} \mathcal{C}^{2}\left|\hat{F}_{y x}\right|^{2}+\beta_{y}^{2} \mathcal{C}^{2}\left|\hat{F}_{x y}\right|^{2}\right. \\
- & \left.\beta_{x} \beta_{y}\left(\mathcal{C}^{4}-4 \mathcal{C}^{2} \Im \hat{F}_{y x} \Im \hat{F}_{x y}+\left|\hat{F}_{y x}\right|^{2}\left|\hat{F}_{x y}\right|^{2}\right)\right],
\end{aligned}
$$

where again the subscript ${ }_{w}$ has been omitted. Unlike the previous case for the tune change, here no general tendency leading to a reduction is observed, with the change being rather dependent on the ratio of the $\beta$-functions. However, for a case like the LHC where the Landau octupoles are positioned next to main quadrupoles in the arc and, as such, the $\beta$-function in one plane is significantly larger than that in the other plane, the direct amplitude detuning term in the focusing plane of the quadrupole as well as the crossterm detuning will decrease.

To benchmark the derived formulas, again studies using a thin LHC lattice as testbed were conducted using MAD-X-PTC. Similar to studies presented in [11], 4 skew quadrupoles were installed in the dispersion suppressor left and right of arc 12 in the LHC to create a closed coupling bump. This is illustrated in Fig. 5. One octupole next to a defocusing quadrupole was then powered to three quarter of its maximum gradient of $6.3 \times 10^{4} \mathrm{~T} / \mathrm{m}^{3}$ and the amplitude detuning with and without the coupling bump was determined. In both cases, all other nonlinear elements have been turned off to avoid contributions from other sources. Tunes were rematched to the original values of $Q_{x}=0.28$ and $Q_{y}=0.31$ after application of the coupling bump using quadrupoles located outside of the bump. The results are presented in Table I showing a good agreement between the derived formula and MAD-X-PTC. To show that this effect is caused by the local coupling, the amplitude detuning generated by another octupole in the neighboring arc 23 is presented in Table II.

The small change in amplitude detuning generated by an octupole in arc 23 between the case of no coupling bump and with a coupling bump applied in arc 12 in Table II is explained by the second order effect of the skew quadrupoles on the optics. Here, the $\beta$-function at the octupole in arc 23 changes by $0.15 \%$ and $-0.09 \%$ in the horizontal and vertical plane, respectively. As for the case of amplitude detuning generated by the octupole in the

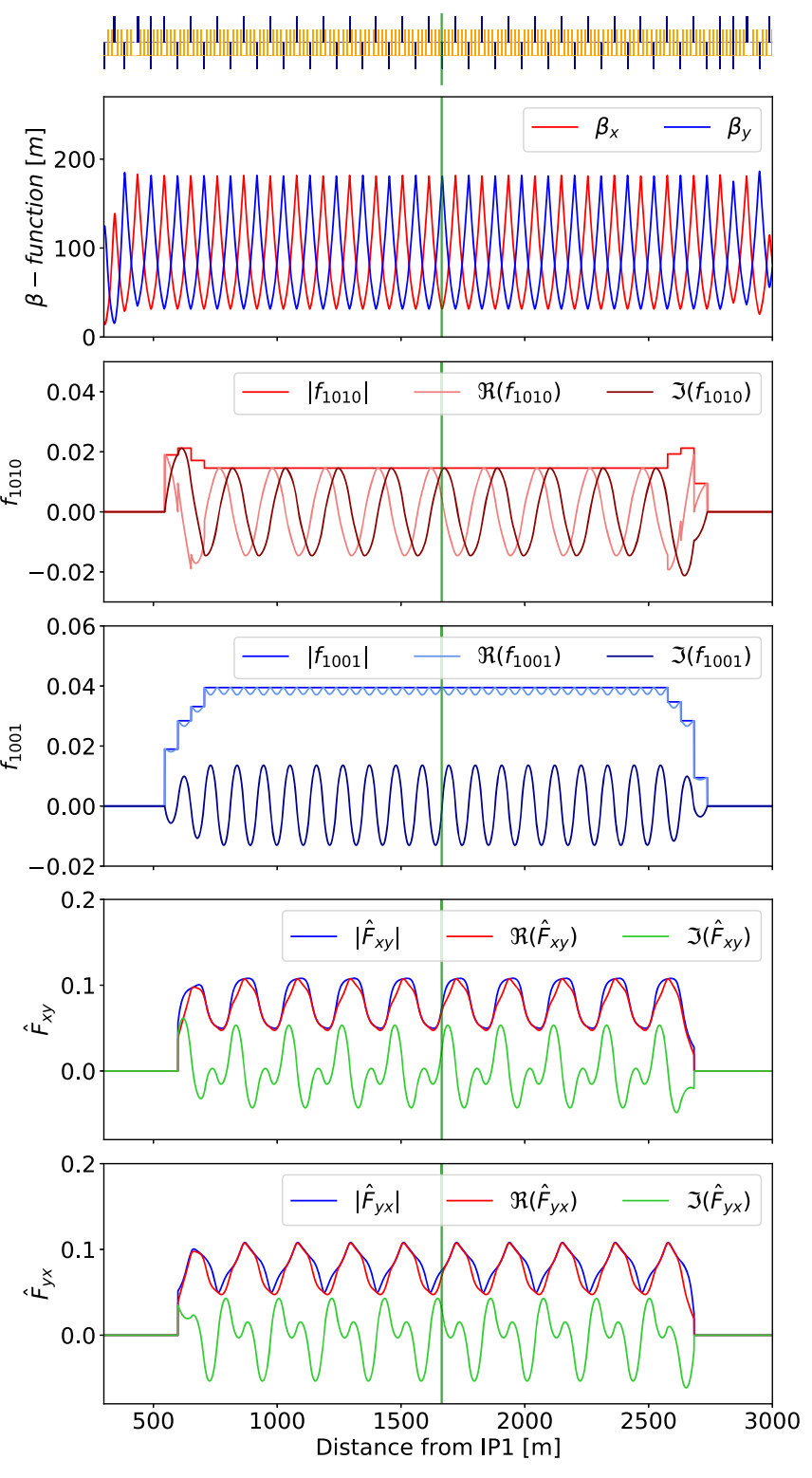

FIG. 5. Illustration of the four corrector coupling bump created through the arc 12 right of IP1. The position of the octupole is indicated with a green line.

coupling bump shown in Table I, small differences between the derived formulas and MAD-X-PTC are observed in the cross term $\left(d Q_{x} / d J_{y}\right)$ and in the direct term of the horizontal plane, which is the plane where the $\beta$-function

TABLE I. Amplitude detuning from a single octupole within the coupling bump in arc 12 .

\begin{tabular}{lrrrrr}
\hline \hline & \multicolumn{2}{c}{ No coupling bump } & & \multicolumn{2}{c}{ With coupling bump } \\
\cline { 2 - 3 } \cline { 5 - 6 } & Formula & PTC & & Formula & PTC \\
\hline$d Q_{x} / d J_{x}$ & $2.16 \times 10^{2}$ & $2.16 \times 10^{2}$ & & $1.98 \times 10^{2}$ & $1.95 \times 10^{2}$ \\
$d Q_{y} / d J_{y}$ & $6.17 \times 10^{3}$ & $6.17 \times 10^{3}$ & & $6.05 \times 10^{3}$ & $6.05 \times 10^{3}$ \\
$d Q_{x} / d J_{y}$ & $-2.31 \times 10^{3}$ & $-2.31 \times 10^{3}$ & & $-2.19 \times 10^{2}$ & $-2.20 \times 10^{2}$ \\
\hline \hline
\end{tabular}


TABLE II. Amplitude detuning from a single octupole outside of the coupling bump in arc 23 .

\begin{tabular}{lrrrrr}
\hline \hline & \multicolumn{2}{c}{ No coupling bump } & & \multicolumn{2}{c}{ With coupling bump } \\
\cline { 2 - 3 } \cline { 5 - 6 } & Formula & PTC & & Formula & \multicolumn{1}{c}{ PTC } \\
\hline$d Q_{x} / d J_{x}$ & $2.16 \times 10^{2}$ & $2.16 \times 10^{2}$ & & $2.11 \times 10^{2}$ & $2.11 \times 10^{2}$ \\
$d Q_{y} / d J_{y}$ & $6.17 \times 10^{3}$ & $6.17 \times 10^{3}$ & & $6.20 \times 10^{3}$ & $6.20 \times 10^{3}$ \\
$d Q_{x} / d J_{y}$ & $-2.31 \times 10^{3}$ & $-2.31 \times 10^{3}$ & $-2.29 \times 10^{3}$ & $-2.29 \times 10^{3}$ \\
\hline \hline
\end{tabular}

is smaller. Using instead an octupole closer to a focusing quadrupole $\left(\beta_{x} \gg \beta_{y}\right)$, the deviation in the horizontal amplitude detuning is reduced while it increases for the detuning with amplitude in the vertical plane. This implies that formula are more accurate for the leading amplitude detuning term. In Table III, a comparison between the analytical derivations and MAD-X-PTC is presented again for amplitude detuning of an octupole close to a defocusing quadrupole but with the amplitude of coupling bump being a factor 5 larger compared to the case presented in Fig. 5. Here the relative error between formulas and MAD-X-PTC is larger for all terms compared to the previous case with again both the cross term and the direct detuning in the horizontal plane showing a larger relative deviation than the leading term $\left(d Q_{y} / d J_{y}\right)$. Notably, for the same level of local coupling, the relative difference between the derived formulas and the results from MAD-X-PTC is not affected by the strength of the octupole. Furthermore, for the given configuration with the closed coupling bump, the relative deviation only changes slightly for working points with a smaller tune separation, suggesting that in the given case the influence of a possible (amplitude-dependent) closest tune approach on the tracking results is negligible. Due to these aforementioned considerations, the derived formulas represent well the change of amplitude detuning in the presence of local linear coupling to leading order and deviation may arise from additional coupling terms which are not accounted for in the presented derivation.

\section{Impact on local RDTs}

In [10], it was not only shown that coupling affects global quantities such as amplitude detuning but also a shift of the amplitude of measured skew sextupole RDTs is observed. As derived in [13] this shift could be partially

TABLE III. Amplitude detuning from a single octupole within the coupling bump in arc 12 with increased levels of local coupling.

\begin{tabular}{lrrrrr}
\hline \hline & \multicolumn{2}{c}{ No coupling bump } & & \multicolumn{2}{c}{ With coupling bump } \\
\cline { 2 - 3 } \cline { 5 - 6 } & Formula & PTC & & Formula & \multicolumn{1}{c}{ PTC } \\
\hline$d Q_{x} / d J_{x}$ & $2.16 \times 10^{2}$ & $2.16 \times 10^{2}$ & & $-4.07 \times 10^{1}$ & $-6.95 \times 10^{1}$ \\
$d Q_{y} / d J_{y}$ & $6.17 \times 10^{3}$ & $6.17 \times 10^{3}$ & & $3.54 \times 10^{3}$ & $3.73 \times 10^{3}$ \\
$d Q_{x} / d J_{y}$ & $-2.31 \times 10^{3}$ & $-2.31 \times 10^{3}$ & & $5.26 \times 10^{1}$ & $-2.19 \times 10^{2}$ \\
\hline \hline
\end{tabular}

explained by taking into account second order contributions from coupling in conjunction with normal sextupoles. However, from these derivations it is not apparent if the skew sextupole RDT would change in the absence of normal sextupoles.

Using the Hamiltonian for a normal sextupole as an example, in the following it is shown how the generated RDTs are affected under local coupling in the absence of other skew multipoles. The coefficients from Eq. (28) in the presence of local linear coupling for the sextupole RDTs $f_{3000}$ and $f_{2100}$ read

$$
h_{w, 3000}^{c}=\frac{K_{w, 3}}{48}\left[\beta_{x}^{\frac{3}{2}} \mathcal{C}^{3}+\sqrt{\beta_{x}} \beta_{y} \mathcal{C}\left|\hat{F}_{x y}\right|^{2}\right]
$$

and

$$
h_{w, 2100}^{c}=\frac{K_{w, 3}}{8}\left[\beta_{x}^{\frac{3}{2}} \mathcal{C}^{3}-\sqrt{\beta_{x}} \beta_{y} \mathcal{C}\left|\hat{F}_{x y}\right|^{2}\right]
$$

In the uncoupled case, both terms stem from the $x^{3}$ term in the sextupole Hamiltonian

$$
H_{3}=\frac{K_{3}}{3 !}\left(x^{3}-3 x y^{2}\right)
$$

whereas in the coupled case an additional contribution from the $x y^{2}$ term is observed.

To benchmark the derived formulas, the same setup was used as in the study for the amplitude detuning. Two sextupoles with a phase difference of $2 \pi$ in both planes
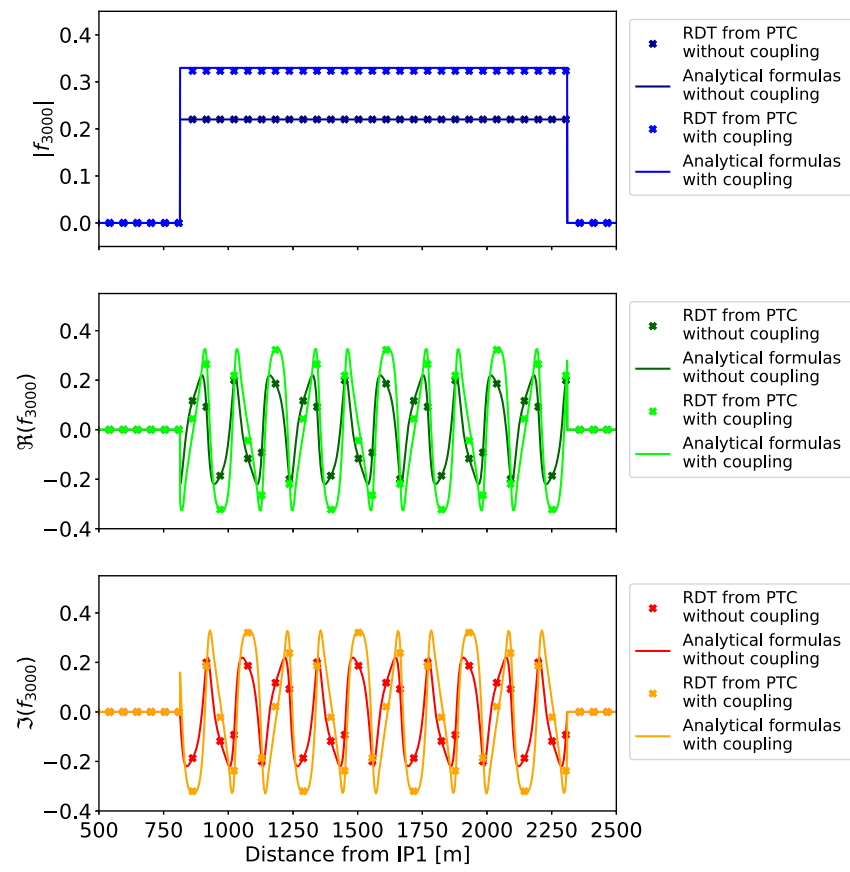

FIG. 6. Comparison for the sextupole RDT $f_{3000}$ between MAD-X-PTC and derived formulas. 

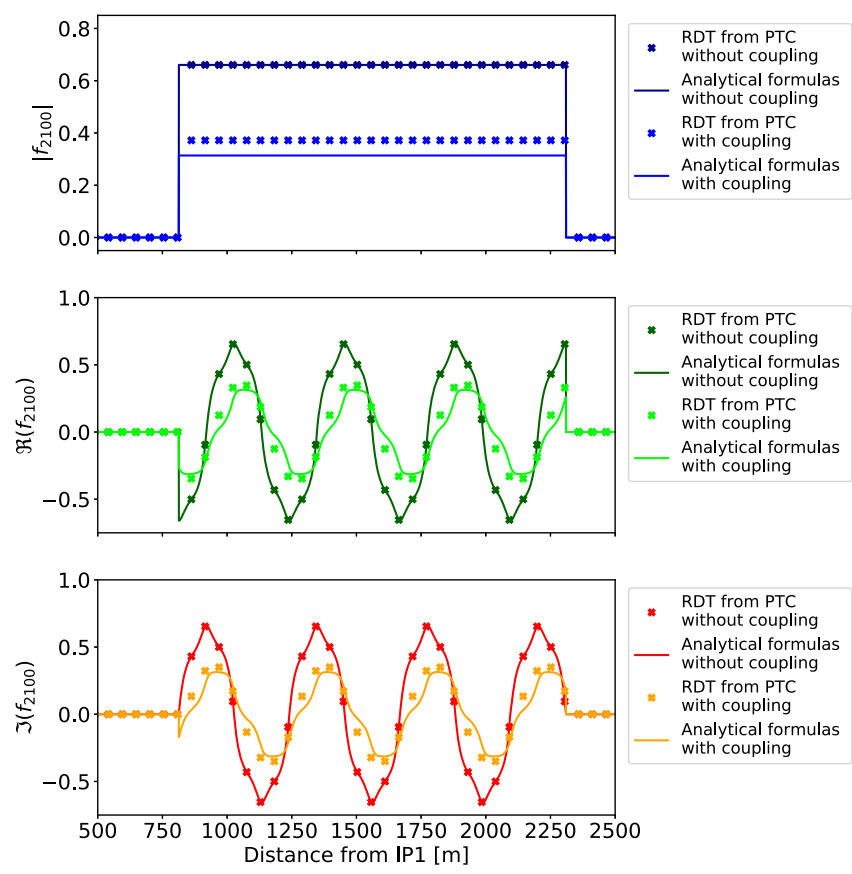

FIG. 7. Comparison for the sextupole RDT $f_{2100}$ between MAD-X-PTC and derived formulas.

were powered to $3 / 4$ of their maximum gradient to create a closed sextupole RDT bump. In Figs. 6 and 7, a comparison between MAD-X-PTC and the analytical formula for the case with and without coupling is presented.

As also in the case of the amplitude detuning, in general a good agreement between the derived formulas and MAD$\mathrm{X}$-PTC is observed. The difference, which is more pronounced for the RDT $f_{2100}$, is again thought to stem from additional coupling terms.

\section{CONCLUSIONS}

Equations relating the impact of local coupling on global and local observables have been presented. While these effects are thought to come from third and higher order contributions, here instead of performing the higher order expansion an alternative approach is presented, using the coupled coordinates in a first order normal form. To illustrate the impact of local linear coupling, the reduction of the amplitude detuning generated by one octupole has been shown for the LHC case as well as the change of resonance driving terms generated by sextupoles. Comparison between the derived analytical formulas and tracking studies show good agreement in the range of interest, with the observed deviations from the tracking code results appearing to stem from additional terms which are not taken into account in the presented approach. The presented formulas also allow us to explain why an erroneous large local coupling in one LHC interaction point did not affect the K-modulation measurements while significantly affecting the beam size and subsequently the luminosity. Given the impact on, e.g., beam size and subsequently possible luminosity decrease, these formulas allow us to establish an upper bound for tolerable local coupling in an accelerator.

\section{ACKNOWLEDGMENTS}

The authors would like to thank Massimo Giovannozzi and Gianluigi Arduini for proofreading the manuscript and providing valuable comments and corrections. Similar thanks go to Ewen Maclean and Tobias Persson for fruitful discussions.

[1] G. Guignard, The general theory of all sum and difference resonances in a three-dimensional magnetic field in a synchrotron, CERN Yellow Reports: Monographs (CERN, Geneva, 1976).

[2] R. Tomás, T. H. B. Persson, and E. H. Maclean, Amplitude dependent closest tune approach, Phys. Rev. Accel. Beams 19, 071003 (2016).

[3] E. H. Maclean, T. Persson, and R. Tomás, Amplitude dependent closest tune approach generated by normal and skew octupoles, in Proc. 8th Int. Particle Accelerator Conf. (IPAC'17), Copenhagen, Denmark, May 2017, paper WEPIK091, pp. 3147-3150, http://jacow.org/ipac2017/ papers/wepik091.pdf, https://doi.org/10.18429/JACoWIPAC2017-WEPIK091, 2017.

[4] T. Persson and R. Tomás, Improved control of the betatron coupling in the Large Hadron Collider, Phys. Rev. Accel. Beams 17, 051004 (2014).

[5] R. Tomás, M. Aiba, A. Franchi, and U. Iriso, Review of linear optics measurement and correction for charged particle accelerators, Phys. Rev. Accel. Beams 20, 054801 (2017).

[6] J. M. Jowett et al., The 2018 Heavy-Ion Run of the LHC, in Proc. IPAC'19, Melbourne, Australia, May 2019 (JACoW, Geneva, 2019), pp. 2258-2261, https://doi.org/10.18429/ JACoW-IPAC2019-WEYYPLM2.

[7] R. Tomás, F. S. Carlier, J. M. C. de Portugal, J. Dilly, S. D. Fartoukh, E. Fol, D. Gamba, A. Garcia-Tabares, M. Giovannozzi, M. Hofer, E. H. Maclean, L. Malina, T. H. B. Persson, P. K. Skowroski, M. Solfaroli, M. L. Spitznagel, A. Wegscheider, J. Wenninger, and D. W. Wolf, LHC Run 2 optics commissioning experience in view of HL-LHC, in Proc. 10th International Particle Accelerator Conference (IPAC'19), Melbourne, Australia, May 2019 (JACoW, Geneva, 2019), pp. 508-511, https://doi.org/10.18429/ JACoW-IPAC2019-MOPMP033.

[8] F. Pilat, Y. Luo, N. Malitsky, and V. Ptitsyn, Beam-Based Non-Linear Optics Corrections in Colliders, in Proceedings of the 21st Particle Accelerator Conference, Knoxville, TN, 2005 (IEEE, Piscataway, NJ, 2005), pp. 601-605.

[9] E. H. Maclean, R. Tomás, M. Giovannozzi, and T. H. B. Persson, First measurement and correction of nonlinear errors in the experimental insertions of the CERN Large Hadron Collider, Phys. Rev. Accel. Beams 18, 121002 (2015). 
[10] E. H. Maclean, F. S. Carlier, M. Giovannozzi, T. Persson, and R. Tomás, Effect of linear coupling on nonlinear observables at the LHC, in Proc. 8th Int. Particle Accelerator Conf. (IPAC'17), Copenhagen, Denmark, May 2017 (JACoW, Genevea, 2017), paper WEPIK092, pp. 3151-3154, http://jacow.org/ipac2017/papers/wepik092 .pdf, https://doi.org/10.18429/JACoW-IPAC2017-WEPIK092, 2017.

[11] L. R. Carver, X. Buffat, K. Li, E. Métral, and M. Schenk, Transverse beam instabilities in the presence of linear coupling in the Large Hadron Collider, Phys. Rev. Accel. Beams 21, 044401 (2018).

[12] E. H. Maclean, R. Tomás, F. S. Carlier, M. S. Camillocci, J. W. Dilly, J. C. de Portugal, E. Fol, K. Fuchsberger, A. G.-T. Valdivieso, M. Giovannozzi, M. Hofer, L. Malina, T. H. B. Persson, P. K. Skowronski, and A. Wegscheider, New approach to LHC optics commissioning for the nonlinear era, Phys. Rev. Accel. Beams 22, 061004 (2019).

[13] A. Franchi, L. Farvacque, F. Ewald, G. Le Bec, and K. B. Scheidt, First simultaneous measurement of sextupolar and octupolar resonance driving terms in a circular accelerator from turn-by-turn beam position monitor data, Phys. Rev. Accel. Beams 17, 074001 (2014).

[14] A. Franchi, E. Métral, and R. Tomás, Emittance sharing and exchange driven by linear betatron coupling in circular accelerators, Phys. Rev. Accel. Beams 10, 064003 (2007).
[15] J. Bengtsson and J. Irwin, Analytical calculations of smear and tune shift, Report No. SSC-232, 1990.

[16] R. Bartolini and F. Schmidt, Normal form via tracking or beam data, Part. Accel. 59, 93 (1998).

[17] R. Calaga, R. Tomás, and A. Franchi, Betatron coupling: Merging Hamiltonian and matrix approaches, Phys. Rev. Accel. Beams 8, 034001 (2005).

[18] M. G. Minty and F. Zimmermann, Measurement and Control of Charged Particle Beams (Springer, Berlin, 2013).

[19] P. Thrane, R. Tomás, A. Koval, K. Ohmi, Y. Ohnishi, and A. Wegscheider, Measuring $\beta^{*}$ in SuperKEKB with $K$ modulation, Phys. Rev. Accel. Beams 23, 012803 (2020).

[20] T. H. B. Persson, F. S. Carlier, M. Hofer, E. H. Maclean, R. Tomás, and A. G.-T. Valdivieso, MD4944: Local linear coupling measurement at the IPs, Report No. CERN-ACCNOTE-2020-0013, 2020.

[21] T. H. B. Persson, Post-mortem analysis of IR2 coupling issue, 372nd LHC machine committee meeting, 2019.

[22] A. Franchi, L. Farvacque, J. Chavanne, F. Ewald, B. Nash, K. Scheidt, and R. Tomás, Vertical emittance reduction and preservation in electron storage rings via resonance driving terms correction, Phys. Rev. Accel. Beams 14, 034002 (2011).

[23] Y. Cai, Luminosity of asymmetric $e^{+} e^{-}$collider with coupling lattices, Report No. SLAC-PUB-8479, 2000. 